\title{
NON-MISCIBILITY AND THE MASS LAW
}

BY WILDER D. BANCROF'T

If we have two liquids $A$ and $B$ reacting to form a compound $\mathrm{AB}$, and if there are no disturbing factors, the reversible equilibrium in the liquid phase should be described by the equation

$$
\mathrm{K} x=y z
$$

where $x, y$, and $z$ are the volume concentrations of the substances $A B, A$ and $B$ respectively. We will now consider the effect of the assumption that $\mathrm{A}$ and $\mathrm{B}$ are non-miscible liquids, each one being miscible in all proportions with the compound AB. This case can be realized approximately with the system chloral hydrate, chloral and water. Above the meltingpoint chloral hydrate is miscible in all proportions with either chloral or water. On the other hand, chloral and water form two liquid layers. I know of no measurements which show to what extent the two are miscible. This is, however, for the moment an unimportant detail.

If the components $\mathrm{A}$ and $\mathrm{B}$ are non-miscible each will make the other less soluble in the mixture and each will therefore increase the chemical potential $\mu$ of the other. ${ }^{1}$ Since the condition for equilibrium ${ }^{2}$ is that

$$
\mu_{x}=\mu_{y}+\mu_{z}
$$

an increase in the chemical potentials of one of the reacting systems will displace the equilibrium and will be equivalent to an increase in the concentrations. Incidentally, it may be mentioned that making $A$ and $B$ non-miscible lowers the vapor pressure $^{3}$ of the compound $\mathrm{AB}$ and therefore decreases the chemical potential $\mu_{x}$. The two effects therefore work in the same direction and the qualitative result of our assumption is a decrease in the effective concentration of the undissociated compound with a resulting forcing-back of the percentage dissociation.

${ }^{1}$ Miller: Jour. Phys. Chem., I, 633 (I907).

2 Gibbs: Thermodynamische Studien, I 12.

${ }^{3}$ McIntosh: Jour. Phys. Chem., I, 474 (Igo7). 
Putting this in another form, we see that making the two components $\mathrm{A}$ and $\mathrm{B}$ non-miscible is equivalent to increasing the apparent values of $y$ and $z$, and to decreasing the apparent value of $x$. If the experimentally determined values of the concentrations are not the values to be used in calculating the equilibrium relations, we may get unexpected and disconcerting results if we do not take this into account.

We do not know as yet, the way in which the chemical potentials of $\mathrm{A}$ and $\mathrm{B}$ are changed quantitatively by the introduction of the phenomenon of non-miscibility, but we can work out the effect of certain arbitrary assumptions. We will let $x, y$, and $z$ be as before the analytically determined concentrations of $\mathrm{AB}, \mathrm{A}$ and $\mathrm{B}$ respectively and we-will let $\mathrm{X}$, $\mathrm{Y}$ and $\mathrm{Z}$ be the theoretical concentrations, due to the assumed non-miscibility of the components A and B. Our equation for equilibrium then becomes:

$$
\mathrm{KX}=\mathrm{YZ} \text {. }
$$

To simplify the problem still further, we will ignore the change in the chemical potential of the compound, which means that we write

$$
x=\mathrm{X} \text {. }
$$

This probably involves only a slight error for cases in which the compound does not dissociate to any great extent when the components $\mathrm{A}$ and $\mathrm{B}$ are present in equivalent quantities.

In the discussion that follows I am considering the chemical potentials of the components in the vapor phase as given by the equations,

$$
\begin{aligned}
& \mu_{\mathrm{A}}=\mathrm{RT}\left(\log p_{a}+\mathrm{C}_{1}\right) \\
& \mu_{\mathrm{B}}=\mathrm{RT}\left(\log p_{\mathrm{B}}+\mathrm{C}_{2}\right) .
\end{aligned}
$$

Case I.-The increase in the chemical potential of either component may be a constant and independent of the absolute concentration of either. If these be true we shall have:

$$
\begin{aligned}
\mathrm{Y} & =k_{1} y \\
\mathrm{Z} & =k_{2} z \\
\frac{\mathrm{K}}{k_{1} k_{2}} x & =y z .
\end{aligned}
$$

Under these circumstances we shall have the same form of equation whether we use the theoretical or the analytical 
concentrations, the only difference being that the equilibrium constant will change from $\mathrm{K}$ to $\mathrm{K} / k_{1} k_{2}$. While this case is hypothetically possible, it is not a case which occurs experimentally. We know that experimentally the partial pressure of one of the components changes continuously with addition of the other component when the two components are nonmiscible.

Case II.-The increase in the chemical potential of either component may be directly proportional to the chemical potential of the other component. If this be true we shall have:

$$
\begin{aligned}
\mathrm{Y} & =y z^{n} \\
\mathrm{Z} & =y^{m} z \\
\mathrm{~K} x & =y^{\mathbf{1}+m} z^{\mathbf{1}+n} .
\end{aligned}
$$

Under these circumstances we shall have an entirely different form of equation. If $m=\mathrm{I}$, for instance, we should conclude that the component had a molecular weight equal to half the formula weight whereas that would not be the case at all. If $m$ and $n$ were fractions, we should get slight variations from the expected formula which we should interpret as due to partial polymerization of the compound, to variations from the gas laws, or to some other natural but inapplicable cause.

While Case $I I$ is a more plausible one than Case $I$, it is not very probable that the change of the vapor pressure of one of the components is independent of the absolute concentration of that component even though it may be approximately true between narrow limits.

Case III.-The increase in the chemical potential of either component may be inversely proportional to the chemical potential of that component and directly proportional to the chemical potential of the other component. If this be true we shall have:

$$
\begin{aligned}
\mathrm{Y} & =\frac{y z^{n}}{y^{p}} . \\
\mathrm{Z} & =\frac{y^{m} z}{z^{q}} . \\
\mathrm{K} x & =y^{1+m-\phi} z^{\mathrm{I}+n-q} .
\end{aligned}
$$

${ }^{1}$ The $\mathrm{K}$ in this equation is not the same as in the preceding one since it contains $m$ and $n$ as exponential functions. 
Since $m, n, p$, and $q$ are constants, Case $I I$ and Case III would both give an equation of the general form:

$$
\mathrm{K} x=y^{a} z^{b}
$$

Where $a=\mathrm{I}+m$ and $b=\mathrm{I}+n$ for Case II while $a=\mathrm{I}+m-p$ and $b=I+n-q$ for Case III. Since the equations for Case II and Case III have the same general form, the same general remarks apply with one exception. Under Case $I I$ the exponents cannot be unity. Under Case $I I I$ it is conceivable that $m=p$ and that $n=q$. If so the equation for equilibrium would have the form

$$
\mathrm{K} x=y z
$$

Under these conditions the effect due to the non-miscibility disappears so far as the concentrations of the components is concerned. Systems coming under this head could be distinguished, however, from systems involving complete miscibility by varying the concentrations over such a range that we should have to drop the simplifying assumption that $x=\mathrm{X}$.

Case $I V$. - The change in the chemical potential of either component is some other function of the chemical potentials of the components than that postulated under Case III. Until one assigns a value to the unknown function, one cannot say what form the equation of equilibrium will take, but it is only under special conditions that the effect due to the non-miscibility of the components will disappear as far as the two components are concerned, and there is no reason to suppose that it will ever disappear if one takes into account the difference between the analytical and the theoretical concentration of the compound.

If we exclude Case I as impossible, we reach the conclusion that the mass law formula, as usually applied, will never describe the equilibrium in a single liquid phase for a dissociating compound when the dissociation products are rion-miscible, though each is miscible in all proportions with the compound.

The case where the components are partially miscible is of course intermediate between the two extremes of complete miscibility and complete non-miscibility. There is therefore 
nothing to be gained by discussing such systems in detail before we have experimental data concerning them.

This matter of obtaining experimental data is a serious one. In chloral hydrate we have a substance which dissociates somewhat in the liquid phase at suitable temperatures. In butyl-chloral hydrate we have a substance which dissociates a good deal more. The problem is how to determine the degree of dissociation with accuracy. So far I have not been able to devise any method of analysis which would be satisfactory. I am publishing this paper, partly in the hope that some one else may display greater ingenuity.

Having shown the necessity for modifying the mass law formula when we postulate non-miscibility in a two-component system, it may be well to consider what may happen in threecomponent systems. The simplest form of this is a dissociating compound for which the mass law applies and a solvent with which one of the components is non-miscible. Let us suppose that the component $A$ is non-miscible with the solvent $S$ and that the solution is so dilute that we may treat the precipitating effect of the solvent as practically constant. If the increase in the chemical potential of the component A is directly proportional to the chemical potential of the solvent and inversely proportional to the chemical potential of component $A$ we shall have

$$
\mathrm{Y}=\frac{k y}{y^{n}}
$$

where $k$ is the factor due to the chemical potential of the solvent. The equation for equilibrium therefore becomes

$$
\mathrm{K} x=k y^{\mathrm{i}-n} z,
$$

and we see that the dissociation is not described by the simple mass law formula.

We can next consider a three-component system in which a metathetical reaction takes place according to the equation

$$
\mathrm{AB}+\mathrm{CD}=\mathrm{AD}+\mathrm{BC} \text {. }
$$

The normal equation for equilibrium will be

$$
\mathrm{K} w x=y z
$$


where $w, x, y$ and $z$ are the volume concentrations of the four constituents. We will now postulate that $\mathrm{AD}$ and $\mathrm{BC}$ are practically non-miscible, and that each is miscible in all proportions with $\mathrm{AB}$ and $\mathrm{BC}$. In that case we shall get special exponents for $y$ and $z$ even without considering $w$ and $x$. In order to consider the changes in $w$ and $x$, we should have to know how the chemical potentials of a varying mixture of $\mathrm{AB}$ and $\mathrm{BC}$ change with varying absolute and relative amounts of $\mathrm{AD}$ and $\mathrm{BC}$. This cannot be done at present, but we see that there will be less formation of $\mathrm{AD}$ and $\mathrm{BC}$ if these substances are non-miscible than would be the case if they were completely miscible. We also see that the simple mass law formula will not describe the equilibrium relations' accurately. To a certain extent the ester formation comes under this head, because ethyl acetate and water are only miscible to a limited extent. It may therefore be asked how it happens that the mass law formula appears to describe the facts so well. The answer is not hard to find. In the first place only a relatively small portion of the equilibrium field has been worked over. In the second place the work has been done either with alcohol or acetic acid in excess, or with water in very large excess. In other words, the experimenters have always kept well away from the saturation point where the deviations from the simple formula would be the most marked. It would not be safe to say that the remarkable results obtained by Price ${ }^{1}$ in his work on the equilibrium between ethyl alcohol and hydrochloric acid were due to limited solubility, but it is worthy of notice that marked discrepancies between theory and experiment do occur in a system where the appearance of a second liquid layer has to be guarded against.

The general result of this investigation is that the simple mass law formula will not describe the equilibrium in any case in which the liquid phase contains two substances which are more or less completely non-miscible.

Cornell University

' Jour. Chem. Soc., 79, 303 (IgOr), 